\title{
Adaptation of Differential Transform Method for the Numeric-Analytic Solution of Fractional-Order Rössler Chaotic and Hyperchaotic Systems
}

\author{
Asad Freihat and Shaher Momani \\ Department of Mathematics, Faculty of Science, The University of Jordan, Amman 1194, Jordan \\ Correspondence should be addressed to Asad Freihat, asadfreihat@yahoo.com
}

Received 20 November 2011; Accepted 15 December 2011

Academic Editor: Muhammad Aslam Noor

Copyright (C) 2012 A. Freihat and S. Momani. This is an open access article distributed under the Creative Commons Attribution License, which permits unrestricted use, distribution, and reproduction in any medium, provided the original work is properly cited.

\begin{abstract}
A new reliable algorithm based on an adaptation of the standard generalized differential transform method (GDTM) is presented. The GDTM is treated as an algorithm in a sequence of intervals (i.e., time step) for finding accurate approximate solutions of fractional-order Rössler chaotic and hyperchaotic systems. A comparative study between the new algorithm and the classical RungeKutta method is presented in the case of integer-order derivatives. The algorithm described in this paper is expected to be further employed to solve similar nonlinear problems in fractional calculus.
\end{abstract}

\section{Introduction}

Mathematical modelling of complex processes is a major challenge for contemporary scientists. In contrast to simple classical systems, where the theory of integer-order differential equations is sufficient to describe their dynamics, complex systems are characterized by the variability of structures in them, multiscale behavior, and nonlinearity in the mathematical description of the mutual relationship between parameters [1]. Recent investigations have shown that many complex physical and biological systems can be represented more accurately through fractional derivative formulation [2]. Fractional derivatives provide an excellent instrument for the description of memory and hereditary properties of various complex materials and systems. Therefore, the number of scientific and engineering problems involving fractional derivatives is already very large and still growing, and perhaps the fractional calculus (i.e., derivatives and integrals of any real or complex order) will be the calculus of the twenty-first century [1-10].

Chaotic systems have a profound effect on its numerical solutions and highly sensitive to time step sizes. It will be beneficial to find a reliable analytical tool to test its longterm accuracy and efficiency. Also, the hyperchaotic systems have more complex dynamical 
behaviors that is because it is defined as a chaotic system with two positive Lyapunov exponents. $\mathrm{Li}$ and Chen [11] have studied the dynamics of both the fractional-order generalizations of the well-known Rössler equation and Rössler hyperchaos equation. They key finding of their study is that chaotic behavior exists in the fractional-order Rössler equation of order as low as 2.4, and hyperchaos exists in the fractional-order Rössler hyperchaos equation of order as low as 3.8. Moaddy et al. [12] used the nonstandard finite difference method to study the dynamic behaviors in the fractional-order Rössler chaotic and hyperchaotic systems. They found that the lowest value to have chaos in this system is 2.1 and hyperchaos exists in the fractional-order Rössler system of order as low as 3.8.

In this paper, we intend to obtain the approximate solution of the fractionalorder Rössler chaotic and hyperchaotic systems via a reliable algorithm based on an adaptation of the generalized differential transform method (GDTM) [13-16], called the multistep generalized differential transform method (MSGDTM). It can be found that the corresponding numerical solutions obtained by using GDTM are valid only for a short time while the ones obtained by using the MSGDTM are more valid and accurate during a long time $[17,18]$.

This paper is organized as follows. In Section 2, we present some necessary definitions and notations related to fractional calculus. In Section 3, the proposed method is described. In Sections 4 and 5, the method is applied to the problems (4.1) and (4.6) and numerical simulations are presented graphically, respectively. Finally, the conclusions are given in Section 6.

\section{Fractional Calculus}

There are several approaches to define the fractional calculus, for example, RiemannLiouville, Grünwald-Letnikov, Caputo, and Generalized Functions approach. RiemannLiouville fractional derivative is mostly used by mathematicians, but this approach is not suitable for real-world physical problems since it requires the definition of fractional order initial conditions, which have no physically meaningful explanation yet, Caputo introduces an alternative definition, which has the advantage of defining integer-order initial conditions for fractional order differential equations.

Definition 2.1. A function $f(x)(x>0)$ is said to be in the space $C_{\alpha}(\alpha \in R)$ if it can be written as $f(x)=x^{p} f_{1}(x)$ for some $p>\alpha$ where $f_{1}(x)$ is continuous in $[0, \infty)$, and it is said to be in the space $C_{\alpha}^{m}$ if $f^{(m)} \in C_{\alpha}, m \in \mathbf{N}$.

Definition 2.2. The Riemann-Liouville integral operator of order $\alpha>0$ with $a \geq 0$ is defined as

$$
\begin{gathered}
\left(J_{a}^{\alpha} f\right)(x)=\frac{1}{\Gamma(\alpha)} \int_{a}^{x}(x-t)^{\alpha-1} f(t) d t, \quad x>a, \\
\left(J_{a}^{0} f\right)(x)=f(x) .
\end{gathered}
$$

Properties of the operator can be found in [3, 19-21]. We only need here the following. For $f \in C_{\alpha}, \alpha, \beta>0, a \geq 0, c \in R$, and $\gamma>-1$, we have

$$
\begin{gathered}
\left(J_{a}^{\alpha} J_{a}^{\beta} f\right)(x)=\left(J_{a}^{\beta} J_{a}^{\alpha} f\right)(x)=\left(J_{a}^{\alpha+\beta} f\right)(x), \\
J_{a}^{\alpha} x^{\gamma}=\frac{x^{\gamma+\alpha}}{\Gamma(\alpha)} B_{(x-a) / x}(\alpha, \gamma+1),
\end{gathered}
$$


where $B_{\tau}(\alpha, \gamma+1)$ is the incomplete beta function which is defined as

$$
\begin{gathered}
B_{\tau}(\alpha, \gamma+1)=\int_{0}^{\tau} t^{\alpha-1}(1-t)^{\gamma} d t \\
J_{a}^{\alpha} e^{c x}=e^{a c}(x-a)^{\alpha} \sum_{k=0}^{\infty} \frac{[c(x-a)]^{k}}{\Gamma(\alpha+k+1)} .
\end{gathered}
$$

The Riemann-Liouville derivative has certain disadvantages when trying to model realworld phenomena with fractional differential equations. Therefore, we shall introduce a modified fractional differential operator $D_{a}^{\alpha}$ proposed by Caputo in his work on the theory of viscoelasticity.

Definition 2.3. The Caputo fractional derivative of $f(x)$ of order $\alpha>0$ with $a \geq 0$ is defined as

$$
\left(D_{a}^{\alpha} f\right)(x)=\left(J_{a}^{m-\alpha} f^{(m)}\right)(x)=\frac{1}{\Gamma(m-\alpha)} \int_{a}^{x} \frac{f^{(m)}(t)}{(x-t)^{\alpha+1-m}} d t
$$

for $m-1<\alpha \leq m, m \in \mathbf{N}, x \geq a, f(x) \in C_{-1}^{m}$.

The Caputo fractional derivative was investigated by many authors; for $m-1<\alpha \leq m$, $f(x) \in C_{\alpha}^{m}$, and $\alpha \geq-1$, we have

$$
\left(J_{a}^{\alpha} D_{a}^{\alpha} f\right)(x)=J^{m} D^{m} f(x)=f(x)-\sum_{k=0}^{m-1} f^{(k)}(a) \frac{(x-a)^{k}}{k !} .
$$

For mathematical properties of fractional derivatives and integrals, one can consult the mentioned references.

\section{Multistep Generalized Differential Transform Method (MSGDTM)}

Although the generalized differential transform method (GDTM) is used to provide approximate solutions for nonlinear problems in terms of convergent series with easily computable components, it has been shown that the approximated solution obtained is not valid for large $t$ for some systems [13-16]. Therefore, we use the multistep generalized differential transform method (MSGDTM), which offers accurate solution over a longer time frame compared to the standard generalized differential transform method [17, 18].

For this purpose, we consider the following initial value problem for systems of fractional differential equations

$$
\begin{aligned}
D_{*}^{\alpha_{1}} y_{1}(t) & =f_{1}\left(t, y_{1}, y_{2}, \ldots, y_{n}\right), \\
D_{*}^{\alpha_{2}} y_{2}(t) & =f_{1}\left(t, y_{1}, y_{2}, \ldots, y_{n}\right), \\
& \vdots \\
D_{*}^{\alpha_{n}} y_{n}(t) & =f_{1}\left(t, y_{1}, y_{2}, \ldots, y_{n}\right),
\end{aligned}
$$


subject to the initial conditions

$$
y_{i}\left(t_{0}\right)=c_{i}, \quad i=1,2, \ldots, n,
$$

where $D_{*}^{\alpha_{i}}$ is the Caputo fractional derivative of order $\alpha_{i}$, where $0<\alpha_{i} \leq 1$, for $i=1,2, \ldots, n$. Let $\left[t_{0}, T\right]$ be the interval over which we want to find the solution of the initial value problem (3.1)-(3.2). In actual applications of the generalized differential transform method (GDTM), the $K$ th-order approximate solution of the initial value problem (3.1)-(3.2) can be expressed by the finite series

$$
y_{i}(t)=\sum_{i=0}^{K} Y_{i}(k)\left(t-t_{0}\right)^{k \alpha_{i}}, \quad t \in\left[t_{0}, T\right]
$$

where $Y_{i}(k)$ satisfied the recurrence relation

$$
\frac{\Gamma\left((k+1) \alpha_{i}+1\right)}{\Gamma\left(k \alpha_{i}+1\right)} Y_{i}(k+1)=F_{i}\left(k, Y_{1}, Y_{2}, \ldots, Y_{n}\right),
$$

$Y_{i}(0)=c_{i}$, and $F_{i}\left(k, Y_{1}, Y_{2}, \ldots, Y_{n}\right)$ is the differential transform of function $f_{i}\left(t, y_{1}, y_{2}, \ldots, y_{n}\right)$ for $i=1,2, \ldots, n$. The basic steps of the GDTM can be found in $[12,15]$.

Assume that the interval $\left[t_{0}, T\right]$ is divided into $M$ subintervals $\left[t_{m-1}, t_{m}\right], m=$ $1,2, \ldots, M$ of equal step size $h=\left(T-t_{0}\right) / M$ by using the nodes $t_{m}=t_{0}+m h$. The main ideas of the MSGDTM are as follows.

First, we apply the GDTM to the initial value problem (3.1)-(3.2) over the interval $\left[t_{0}, t_{1}\right]$, we will obtain the approximate solution $y_{i, 1}(t), t \in\left[t_{0}, t_{1}\right]$, using the initial condition $y_{i}\left(t_{0}\right)=c_{i}$, for $i=1,2, \ldots, n$. For $m \geq 2$ and at each subinterval $\left[t_{m-1}, t_{m}\right]$, we will use the initial condition $y_{i, m}\left(t_{m-1}\right)=y_{i, m-1}\left(t_{m-1}\right)$ and apply the GDTM to the initial value problem (3.1)-(3.2) over the interval $\left[t_{m-1}, t_{m}\right]$. The process is repeated and generates a sequence of approximate solutions $y_{i, m}(t), m=1,2, \ldots, M$, for $i=1,2, \ldots, n$. Finally, the MSGDTM assumes the following solution:

$$
y_{i}(t)= \begin{cases}y_{i, 1}(t), & t \in\left[t_{0}, t_{1}\right] \\ y_{i, 2}(t), & t \in\left[t_{1}, t_{2}\right] \\ \vdots & \\ y_{i, M}(t), & t \in\left[t_{M-1}, t_{M}\right] .\end{cases}
$$

The new algorithm, MSGDTM, is simple for computational performance for all values of $h$. As we will see in the next section, the main advantage of the new algorithm is that the obtained solution converges for wide time regions.

\section{Solving the Fractional-Order Rössler Chaotic and Hyperchaotic Systems Using the MSGDTM Algorithm}

To demonstrate the effectiveness of this scheme, we consider two kinds of systems: the fractional-order Rössler chaotic and hyperchaotic systems. These examples are considered 
because approximate numerical solutions are available for them using other numerical schemes. This allows one to compare the results obtained using this scheme with solutions obtained using other schemes.

\subsection{The Fractional-Order Rössler Chaotic System}

Consider a fractional-order generalization of the Rössler [22]. In this system, the integer-order derivatives are replaced by the fractional-order derivatives, as follows:

$$
\begin{aligned}
& D^{\alpha_{1}} x(t)=-y-z \\
& D^{\alpha_{2}} y(t)=x+a y \\
& D^{\alpha_{3}} z(t)=b+z(x-c)
\end{aligned}
$$

where $(x, y, z)$ are the state variables, $(a, b, c)$ are positive constants, and $\alpha_{i}, i=1,2,3$, are parameters describing the order of the fractional time-derivatives in the Caputo sense. The general response expression contains parameters describing the order of the fractional derivatives that can be varied to obtain various responses. Obviously, the classical integerorder Rössler system can be viewed as a special case from the fractional-order system by setting $\alpha_{1}=\alpha_{2}=\alpha_{3}=1$, which chaotic when $a=0.15$, and for the fractional case, the parameter $a$ is allowed to be varied. In other words, the ultimate behavior of the fractional system response must converge to the response of the integer-order version of the equation.

Applying the MSGDTM algorithm to (4.1) gives

$$
\begin{gathered}
X(k+1)=\frac{\Gamma\left(\alpha_{1} k+1\right)}{\Gamma\left(\alpha_{1}(k+1)+1\right)}(-Y(k)-Z(k)), \\
Y(k+1)=\frac{\Gamma\left(\alpha_{2} k+1\right)}{\Gamma\left(\alpha_{2}(k+1)+1\right)}(X(k)+a Y(k)), \\
Z(k+1)=\frac{\Gamma\left(\alpha_{3} k+1\right)}{\Gamma\left(\alpha_{3}(k+1)+1\right)}\left[b \delta(k)+\left(\sum_{l=0}^{k} Z(l) X(k-l)\right)-c Z(k)\right],
\end{gathered}
$$

where $X(k), Y(k)$, and $Z(k)$ are the differential transformation of $x(t), y(t)$, and $z(t)$, respectively, and $\delta(k)$ equals 1 when $k=0$ and equals 0 otherwise. The differential transform of the initial conditions is given by $X(0)=c_{1}, Y(0)=c_{2}, Z(0)=c_{3}$. In view of the differential inverse transform, the differential transform series solution for the system (4.1) can be obtained as

$$
\begin{aligned}
& X(t)=\sum_{n=0}^{N} X(n) t^{\alpha_{1} n}, \\
& Y(t)=\sum_{n=0}^{N} Y(n) t^{\alpha_{2} n}, \\
& Z(t)=\sum_{n=0}^{N} Z(n) t^{\alpha_{3} n} .
\end{aligned}
$$


According to the multistep generalized differential transform method, the series solution for the system (4.1) is suggested by

$$
\begin{gathered}
X(t)= \begin{cases}\sum_{n=0}^{K} X_{1}(n) t^{\alpha_{1} n}, & t \in\left[0, t_{1}\right], \\
\sum_{n=0}^{K} X_{2}(n)\left(t-t_{1}\right)^{\alpha_{1} n}, & t \in\left[t_{1}, t_{2}\right], \\
\vdots & \\
\sum_{n=0}^{K} X_{M}(n)\left(t-t_{M-1}\right)^{\alpha_{1} n}, & t \in\left[t_{M-1}, t_{M}\right],\end{cases} \\
Y(t)= \begin{cases}\sum_{n=0}^{K} Y_{1}(n) t^{\alpha_{2} n}, & t \in\left[0, t_{1}\right], \\
\sum_{n=0}^{K} Y_{2}(n)\left(t-t_{1}\right)^{\alpha_{2} n}, & t \in\left[t_{1}, t_{2}\right], \\
\vdots & \\
\sum_{n=0}^{K} Y_{M}(n)\left(t-t_{M-1}\right)^{\alpha_{2} n}, & t \in\left[t_{M-1}, t_{M}\right],\end{cases} \\
Z(t)= \begin{cases}\sum_{n=0}^{K} Z_{1}(n) t^{\alpha_{3} n}, & t \in\left[0, t_{1}\right], \\
\sum_{n=0}^{K} Z_{2}(n)\left(t-t_{1}\right)^{\alpha_{3} n}, & t \in\left[t_{1}, t_{2}\right], \\
\vdots & t \in\left[t_{M-1}, t_{M}\right], \\
\sum_{n=0}^{K} Z_{M}(n)\left(t-t_{M-1}\right)^{\alpha_{3} n},\end{cases}
\end{gathered}
$$

where $X_{i}(n), Y_{i}(n)$, and $Z_{i}(n)$ for $i=1,2, \ldots, M$ satisfy the following recurrence relations:

$$
\begin{gathered}
X_{i}(k+1)=\frac{\Gamma\left(\alpha_{1} k+1\right)}{\Gamma\left(\alpha_{1}(k+1)+1\right)}\left(-Y_{i}(k)-Z_{i}(k)\right), \\
Y_{i}(k+1)=\frac{\Gamma\left(\alpha_{2} k+1\right)}{\Gamma\left(\alpha_{2}(k+1)+1\right)}\left(X_{i}(k)+a Y_{i}(k)\right), \\
Z_{i}(k+1)=\frac{\Gamma\left(\alpha_{3} k+1\right)}{\Gamma\left(\alpha_{3}(k+1)+1\right)}\left[b \delta(k)+\left(\sum_{l=0}^{k} Z_{i}(l) X_{i}(k-l)\right)-c Z_{i}(k)\right],
\end{gathered}
$$

such that $X_{i}(0)=x_{i}\left(t_{i-1}\right)=x_{i-1}\left(t_{i-1}\right), Y_{i}(0)=y_{i}\left(t_{i-1}\right)=y_{i-1}\left(t_{i-1}\right)$, and $Z_{i}(0)=z_{i}\left(t_{i-1}\right)=$ $z_{i-1}\left(t_{i-1}\right)$.

Finally, we start with $X_{0}(0)=c_{1}, Y_{0}(0)=c_{2}$, and $Z_{0}(0)=c_{3}$, using the recurrence relation given in (4.5), then we can obtain the multistep solution given in (4.4). 


\subsection{The Fractional-Order Rössler Hyperchaotic System}

The fractional-order Rössler hyperchaotic system [23] is given by

$$
\begin{aligned}
& D^{\alpha_{1}} x(t)=-y-z, \\
& D^{\alpha_{2}} y(t)=x+a y+w, \\
& D^{\alpha_{3}} z(t)=b+x z, \\
& D^{\alpha_{4}} w(t)=c z+d w,
\end{aligned}
$$

where $(x, y, z, w)$ are the state variables, $(a, b, c, d)$ are positive constants, and $\alpha_{i}, i=1,2,3,4$ are parameters describing the order of the fractional time-derivatives in the Caputo sense. In the case of $\alpha_{i}=1(i=1,2,3,4)$, the fractional system reduces to the classical Rössler hyperchaotic system, and it exhibits a hyperchaotic behavior when $a=0.25, b=3, c=0.5$, and $d=0.05$.

Following the same procedure as the previous system and applying MSGDTM algorithm to (4.6) yield

$$
\begin{gathered}
X(k+1)=\frac{\Gamma\left(\alpha_{1} k+1\right)}{\Gamma\left(\alpha_{1}(k+1)+1\right)}(-Y(k)-Z(k)), \\
Y(k+1)=\frac{\Gamma\left(\alpha_{2} k+1\right)}{\Gamma\left(\alpha_{2}(k+1)+1\right)}(X(k)+a Y(k)+W(k)), \\
Z(k+1)=\frac{\Gamma\left(\alpha_{3} k+1\right)}{\Gamma\left(\alpha_{3}(k+1)+1\right)}\left[b \delta(k)+\left(\sum_{l=0}^{k} X(l) Z(k-l)\right)\right], \\
W(k+1)=\frac{\Gamma\left(\alpha_{4} k+1\right)}{\Gamma\left(\alpha_{4}(k+1)+1\right)}(-c Z(k)+d W(k)),
\end{gathered}
$$

where $X(k), Y(k), Z(k)$, and $W(k)$ are the differential transformation of $x(t), y(t), z(t)$, and $w(t)$, respectively. The differential transform of the initial conditions is given by $X(0)=c_{1}$, $Y(0)=c_{2}, Z(0)=c_{3}$, and $W(0)=c_{4}$. In view of the differential inverse transform, the differential transform series solution for the system (4.6) can be obtained as

$$
\begin{aligned}
X(t) & =\sum_{n=0}^{N} X(n) t^{\alpha_{1} n}, \\
Y(t) & =\sum_{n=0}^{N} Y(n) t^{\alpha_{2} n}, \\
Z(t) & =\sum_{n=0}^{N} Z(n) t^{\alpha_{3} n}, \\
W(t) & =\sum_{n=0}^{N} W(n) t^{\alpha_{4} n} .
\end{aligned}
$$


Now, according to the MSGDTM algorithm, the series solution for the system (4.6) is suggested by

$$
\begin{aligned}
& X(t)= \begin{cases}\sum_{n=0}^{K} X_{1}(n) t^{\alpha_{1} n}, & t \in\left[0, t_{1}\right], \\
\sum_{n=0}^{K} X_{2}(n)\left(t-t_{1}\right)^{\alpha_{1} n}, & t \in\left[t_{1}, t_{2}\right], \\
\vdots & \\
\sum_{n=0}^{K} X_{M}(n)\left(t-t_{M-1}\right)^{\alpha_{1} n}, & t \in\left[t_{M-1}, t_{M}\right],\end{cases} \\
& Y(t)= \begin{cases}\sum_{n=0}^{K} Y_{1}(n) t^{\alpha_{2} n}, & t \in\left[0, t_{1}\right], \\
\sum_{n=0}^{K} Y_{2}(n)\left(t-t_{1}\right)^{\alpha_{2} n}, & t \in\left[t_{1}, t_{2}\right], \\
\vdots & \\
\sum_{n=0}^{K} Y_{M}(n)\left(t-t_{M-1}\right)^{\alpha_{2} n}, & t \in\left[t_{M-1}, t_{M}\right],\end{cases} \\
& Z(t)= \begin{cases}\sum_{n=0}^{K} Z_{1}(n) t^{\alpha_{3} n}, & t \in\left[0, t_{1}\right], \\
\sum_{n=0}^{K} Z_{2}(n)\left(t-t_{1}\right)^{\alpha_{3} n}, & t \in\left[t_{1}, t_{2}\right], \\
\vdots & \\
\sum_{n=0}^{K} Z_{M}(n)\left(t-t_{M-1}\right)^{\alpha_{3} n}, & t \in\left[t_{M-1}, t_{M}\right],\end{cases} \\
& W(t)= \begin{cases}\sum_{n=0}^{K} W_{1}(n) t^{\alpha_{3} n}, & t \in\left[0, t_{1}\right], \\
\sum_{n=0}^{K} W_{2}(n)\left(t-t_{1}\right)^{\alpha_{3} n}, & t \in\left[t_{1}, t_{2}\right], \\
\vdots & \\
\sum_{n=0}^{K} W_{M}(n)\left(t-t_{M-1}\right)^{\alpha_{3} n}, & t \in\left[t_{M-1}, t_{M}\right],\end{cases}
\end{aligned}
$$

where $X_{i}(n), Y_{i}(n), Z_{i}(n)$, and $W_{i}(n)$ for $i=1,2, \ldots, M$ satisfy the following recurrence relations:

$$
\begin{aligned}
& X_{i}(k+1)=\frac{\Gamma\left(\alpha_{1} k+1\right)}{\Gamma\left(\alpha_{1}(k+1)+1\right)}\left(-Y_{i}(k)-Z_{i}(k)\right), \\
& Y(k+1)=\frac{\Gamma\left(\alpha_{2} k+1\right)}{\Gamma\left(\alpha_{2}(k+1)+1\right)}\left(X_{i}(k)+a Y_{i}(k)\right),
\end{aligned}
$$




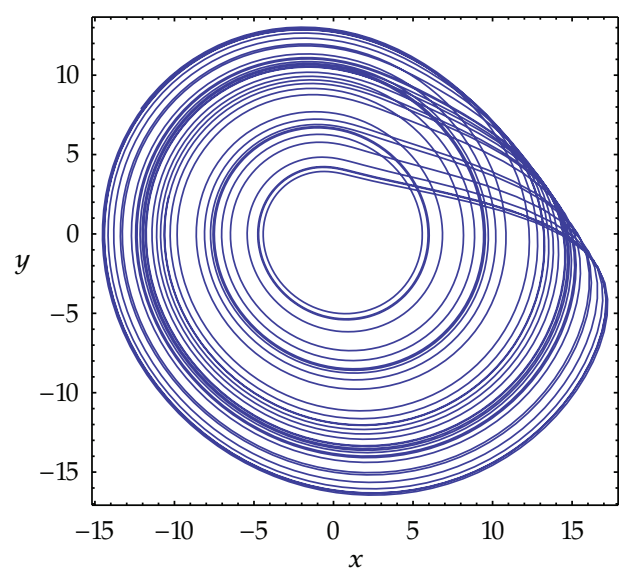

(a)

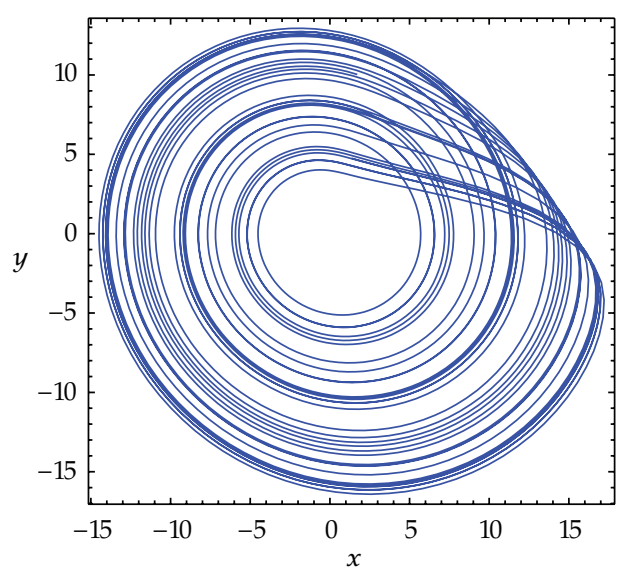

(b)

Figure 1: Phase plot of chaotic attractor in the $x$-y space, with $a=0.15$ and $\alpha_{1}=\alpha_{2}=\alpha_{3}=1$ : (a) MSGDTM, (b) RK4.

$$
\begin{aligned}
& Z_{i}(k+1)=\frac{\Gamma\left(\alpha_{3} k+1\right)}{\Gamma\left(\alpha_{3}(k+1)+1\right)}\left[b \delta(k)+\left(\sum_{l=0}^{k} Z_{i}(l) X_{i}(k-l)\right)-c Z_{i}(k)\right], \\
& W_{i}(k+1)=\frac{\Gamma\left(\alpha_{4} k+1\right)}{\Gamma\left(\alpha_{4}(k+1)+1\right)}\left(-c Z_{i}(k)+d W_{i}(k)\right),
\end{aligned}
$$

such that $X_{i}(0)=x_{i}\left(t_{i-1}\right)=x_{i-1}\left(t_{i-1}\right), Y_{i}(0)=y_{i}\left(t_{i-1}\right)=y_{i-1}\left(t_{i-1}\right), Z_{i}(0)=z_{i}\left(t_{i-1}\right)=z_{i-1}\left(t_{i-1}\right)$, and $W_{i}(0)=w_{i}\left(t_{i-1}\right)=w_{i-1}\left(t_{i-1}\right)$.

Starting with $X_{0}(0)=c_{1}, Y_{0}(0)=c_{2}, Z_{0}(0)=c_{3}, W_{0}(0)=c_{4}$ and using the recurrence relation given in (4.10), we can obtain the multistep solution given in (4.9).

\section{Numerical Results}

We shall demonstrate the accuracy of the MSGDTM against the Mathemtica's built-in fourth-order Runge-Kutta (RK4) procedure for the solutions of both Rössler chaotic and hyperchaotic systems in the case of integer-order derivatives. The MSGDTM is coded in the computer algebra package Mathematica. The Mathematica environment variable digits controlling the number of significant digits are set to 20 in all the calculations done in this paper. The time range studied in this work is $[0,200]$ and the step size $\Delta t=0.05$.

Figures 1 and 2 show the phase portrait for the classical Rössler chaotic system using the multistep generalized differential transform method and the fourth-order RungeKutta method (RK4). From the graphical results in Figures 1 and 2, it can be seen the results obtained using the multistep generalized differential transform method match the results of the RK4 very well, which implies that the multistep generalized differential transform method can predict the behaviour of these variables accurately for the region under consideration. Figures 3 and 4 show the phase portrait for the fractional Rössler chaotic system using the multistep generalized differential transform method. From the numerical results in Figures 3 and 4, it is clear that the approximate solutions depend continuously on 


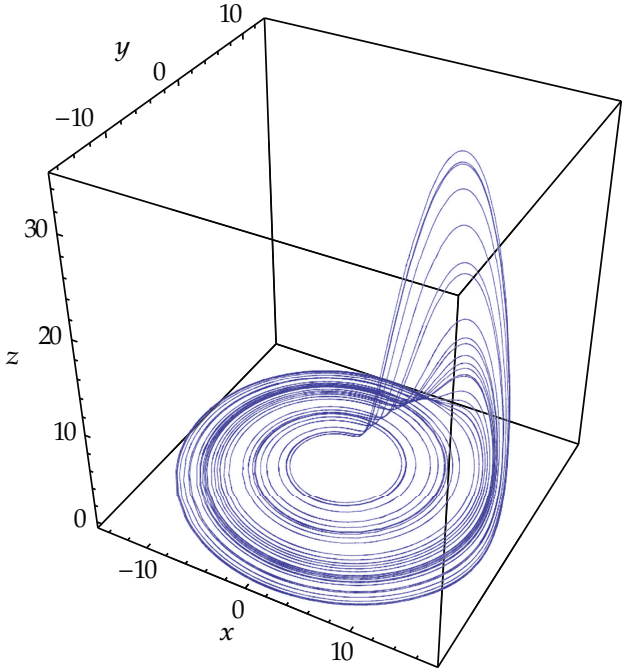

(a)

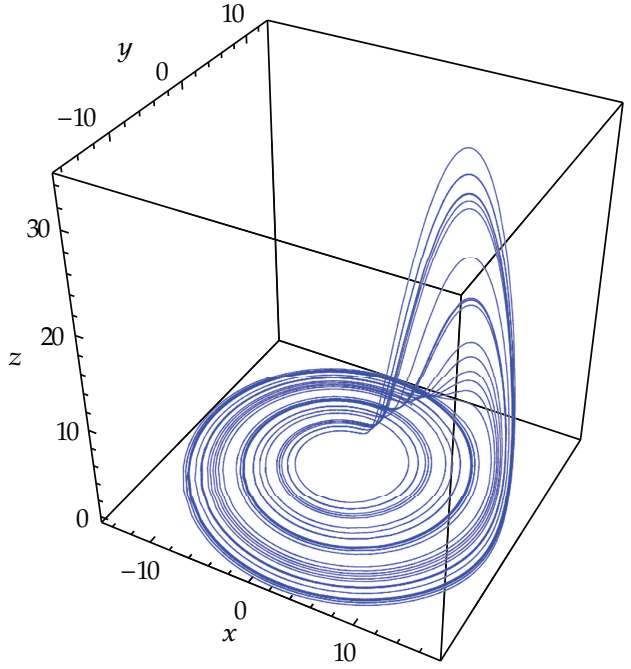

(b)

Figure 2: Phase plot of chaotic attractor in the $x-y$-z space, with $a=0.15$ and $\alpha_{1}=\alpha_{2}=\alpha_{3}=1$ : (a) MSGDTM, (b) RK4.

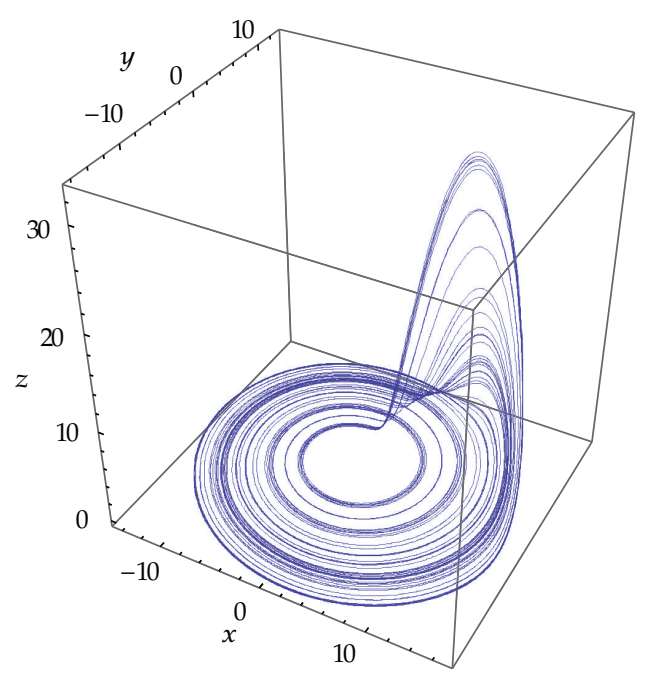

(a)

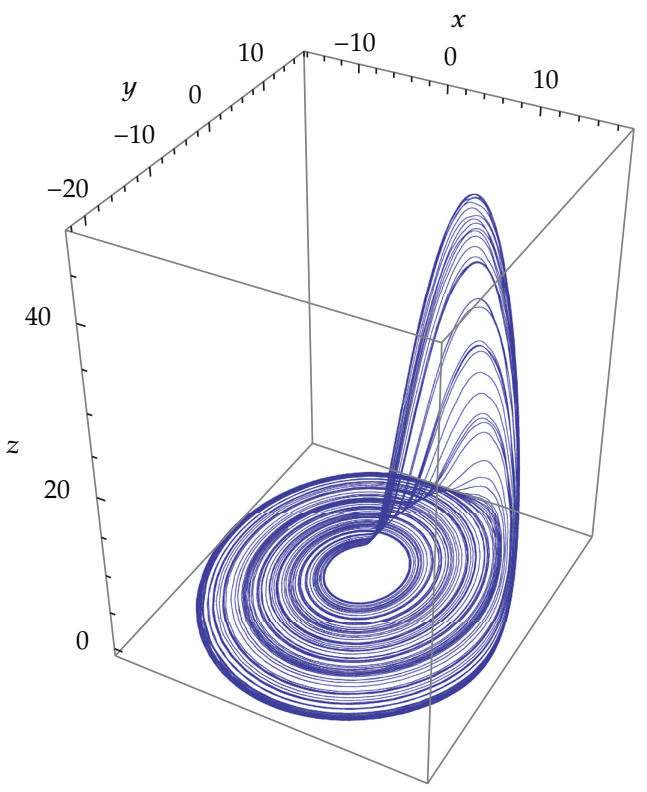

(b)

Figure 3: Phase plot of chaotic attractor in the $x-y-z$ space, with $a=0.15, b=0.2, c=10$ : (a) $\alpha_{1}=\alpha_{2}=$ $\alpha_{3}=0.9,(\mathrm{~b}) \alpha_{1}=0.8, \alpha_{2}=0.7, \alpha_{3}=0.8$.

the time-fractional derivative $\alpha_{i}, i=1,2,3$. The effective dimension $\Sigma$ of (4.1) is defined as the sum of orders $\alpha_{1}+\alpha_{2}+\alpha_{3}=\Sigma$. We can see that the chaos exists in the fractional-order Rössler system with order as low as 2.0 as shown in Figure 4.

Simulations were performed for the classical integer-order Rössler hyperchaotic system in Figure 5. This system has a hyperchaotic attractor when $a=0.25$. Figure 6 shows 


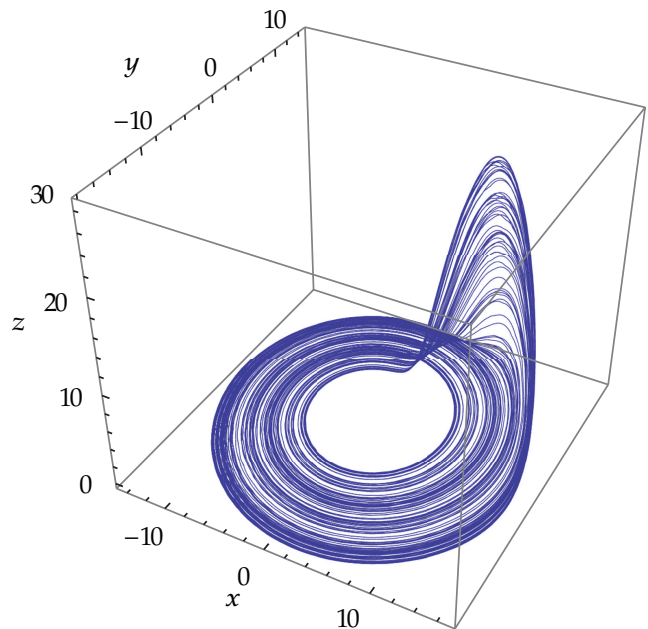

(a)

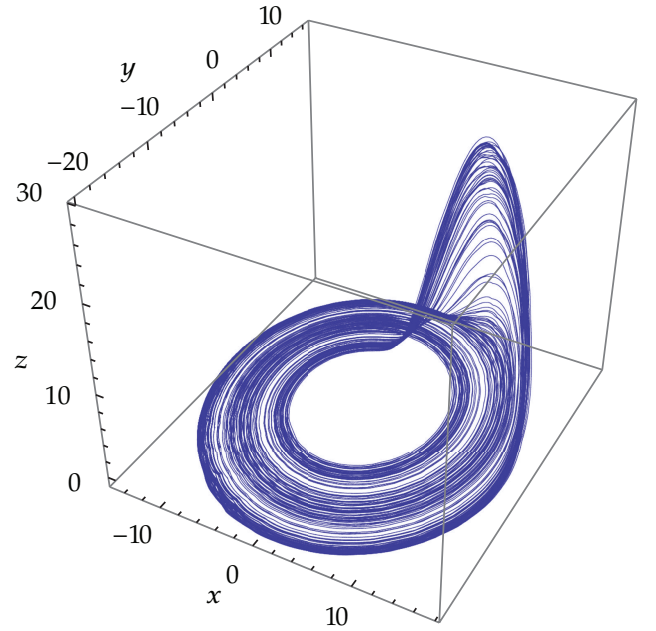

(b)

Figure 4: Phase plot of chaotic attractor in the $x-y-z$ space, with $a=0.15, b=0.2, c=10$ : (a) $\alpha_{1}=0.71$, $\alpha_{2}=0.66, \alpha_{3}=0.71$, (b) $\alpha_{1}=0.69, \alpha_{2}=0.60, \alpha_{3}=0.71$.

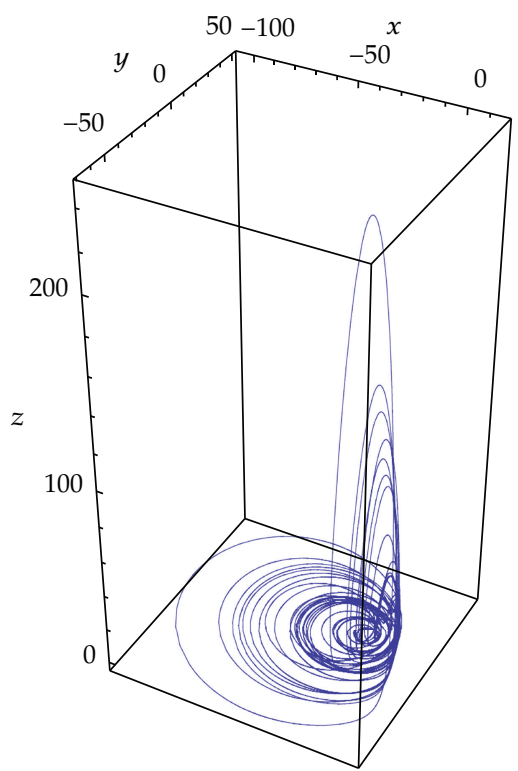

(a)

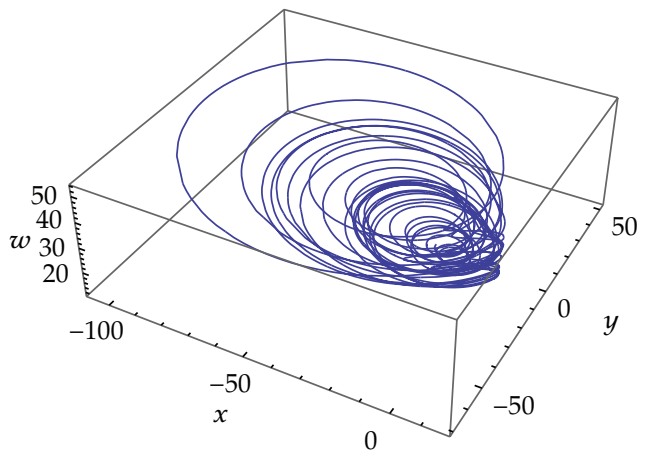

(b)

Figure 5: Phase plot of hyperchaotic attractor in the $x-y-z$ and $x-y-w$ spaces, with $a=0.25, b=3, c=0.5$, $d=0.05$, and $\alpha_{1}=\alpha_{2}=\alpha_{3}=\alpha_{3}=1$.

that the hyperchaos exists in the fractional-order Rössler hyperchaotic system with order as low as 3.64 . 


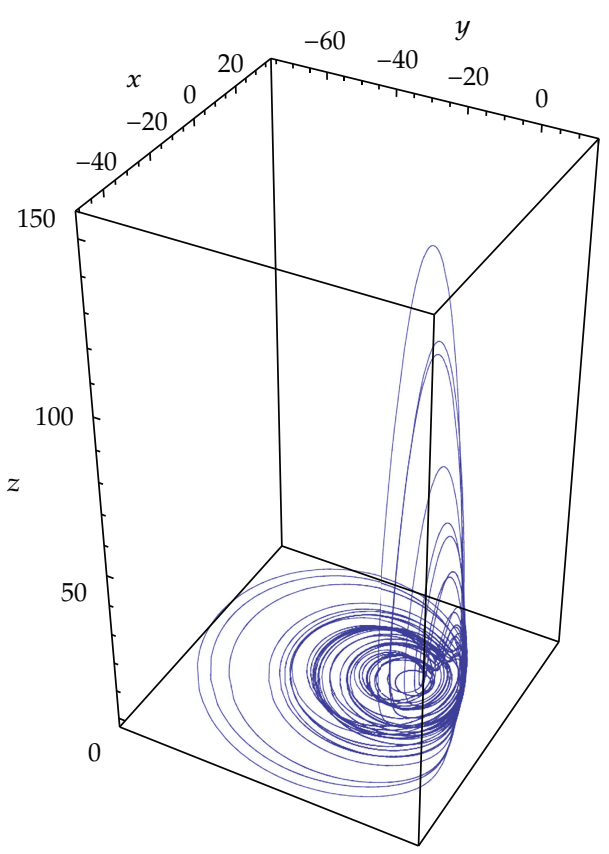

(a)

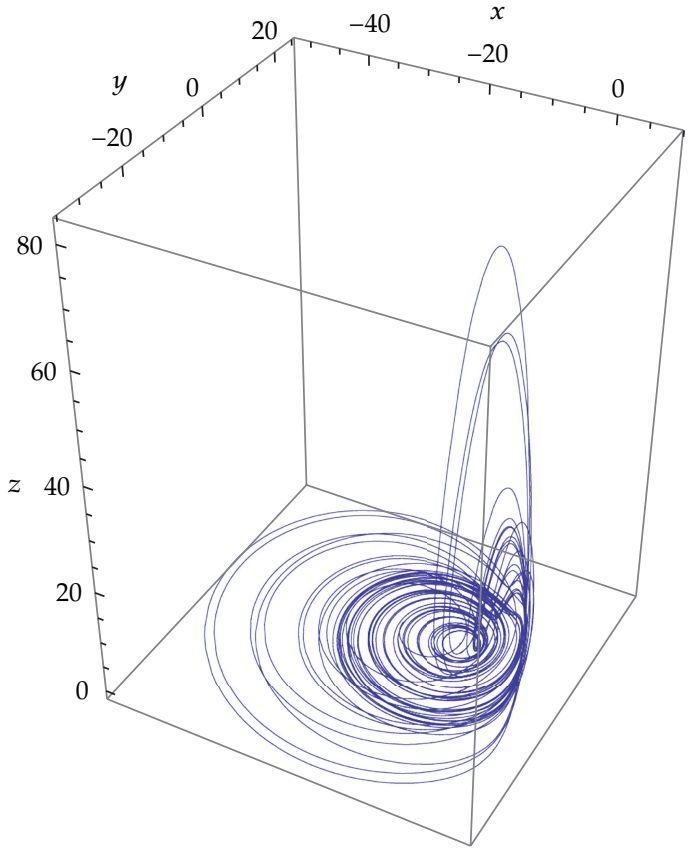

(b)

Figure 6: Phase plot of chaotic attractor in $x-y-z$ spaces, with $a=0.25, b=3, c=0.5, d=0.05$ : (a) $\alpha_{1}=\alpha_{2}=0.98, \alpha_{3}=\alpha_{4}=0.99$, (b) $\alpha_{1}=0.9, \alpha_{2}=0.89, \alpha_{3}=0.88, \alpha_{4}=0.99$.

\section{Conclusions}

In this paper, the multistep generalized differential transform method was utilized successfully to find accurate approximate solutions of fractional-order Rössler chaotic and hyperchaotic systems. The approximate solutions obtained by MSGDTM are highly accurate and valid for a long time. The reliability of the method and the reduction in the size of computational domain give this method a wider applicability. Many of the results obtained in this paper can be extended to significantly more general classes of linear and nonlinear differential equations of fractional order.

\section{References}

[1] J. S. Leszczynski, An Introduction to Fractional Mechanics, Czestochowa University of Technology, Czȩstochowa, Poland, 2011.

[2] F. Mainardi, "Fractional calculus: some basic problems in continuum and statistical mechanics," in Fractals and Fractional Calculus in Continuum Mechanics, A. Carpinteri and F. Mainardi, Eds., pp. 291348, Springer, New York, NY, USA, 1997.

[3] M. Caputo, "Linear models of dissipation whose Q is almost frequency independent-II," Geophysical Journal of the Royal Astronomical Society, vol. 13, no. 5, pp. 529-539, 1967.

[4] A. A. Kilbas, H. M. Srivastava, and J. J. Trujillo, Theory and Applications of Fractional Differential Equations, Elsevier, Amsterdam, The Netherlands, 2006.

[5] R. Hilfer, Applications of Fractional Calculus in Physics, World Scientific, New Jersey, NJ, USA, 2000.

[6] R. L. Bagley and P. J. Torvik, "A theoretical basis for the application of fractional calculus," Journal of Rheology, vol. 27, no. 3, pp. 201-210, 1983. 
[7] R. L. Bagley and R. A. Calico, "Calico. Fractional order state equations for the control of viscoelastically damped structures," Journal of Guidance, Control, and Dynamics, vol. 14, no. 2, pp. 304311, 1991.

[8] E. J. S. Solteiro Pires, J. A. T. Machado, and P. B. de Moura Oliveira, "Fractional order dynamics in a GA planner," Signal Processing, vol. 83, no. 11, pp. 2377-2386, 2003.

[9] K. S. Hedrih and V. N. Stanojevi, "A model of gear transmission: fractional order system dynamics," Mathematical Problems in Engineering, vol. 2010, Article ID 972873, 2010.

[10] J. Cao, C. Ma, H. Xie, and Z. Jiang, "Nonlinear dynamics of duffing system with fractional order damping," Journal of Computational and Nonlinear Dynamics, vol. 5, no. 4, pp. 041012-041018, 2010.

[11] C. P. Li and G. Chen, "Chaos and hyperchaos in the fractional-order Rössler equations," Physica A, vol. 341, no. 1-4, pp. 55-61, 2004.

[12] K. Moaddy, S. Momani, and I. Hashim, "Non-standard finite difference schemes for solving fractional-order Rössler chaotic and hyperchaotic system, ," Computers $\mathcal{E}$ Mathematics with Applications, vol. 61, no. 4, pp. 1209-1216, 2011.

[13] Z. Odibat, S. Momani, and V. S. Erturk, "Generalized differential transform method: application to differential equations of fractional order," Applied Mathematics and Computation, vol. 197, no. 2, pp. 467-477, 2008.

[14] S. Momani and Z. Odibat, "A novel method for nonlinear fractional partial differential equations: combination of DTM and generalized Taylor's formula," Journal of Computational and Applied Mathematics, vol. 220, no. 1-2, pp. 85-95, 2008.

[15] Z. Odibat and S. Momani, "A generalized differential transform method for linear partial differential equations of fractional order," Applied Mathematics Letters, vol. 21, no. 2, pp. 194-199, 2008.

[16] V. S. Erturk, S. Momani, and Z. Odibat, "Application of generalized differential transform method to multi-order fractional differential equations," Communications in Nonlinear Science and Numerical Simulation, vol. 13, no. 8, pp. 1642-1654, 2008.

[17] Z. Odibat, C. Bertelle, M. A. Aziz-Alaoui, and G. Duchamp, "A multi-step differential transform method and application to non-chaotic or chaotic systems," Computers E Mathematics with Applications, vol. 59, no. 4, pp. 1462-1472, 2010.

[18] V. S. Ertürk, Z. Odibat, and S. Momani, “An approximate solution of a fractional order differential equation model of human T-cell lymphotropic virus I (HTLV-I) infection of CD4 ${ }^{+}$T-cells," Computers E Mathematics with Applications, vol. 62, no. 3, pp. 996-1002, 2011.

[19] I. Podlubny, Fractional Differential Equations, vol. 198, Academic Press Inc., San Diego, CA, USA, 1999.

[20] S. Miller and B. Ross, An Introduction to the Fractional Calculus and Fractional Differential Equations, John Wiley \& Sons, New York, NY, USA, 1993.

[21] J. T. Machado, V. Kiryakova, and F. Mainardi, "Recent history of fractional calculus," Communications in Nonlinear Science and Numerical Simulation, vol. 16, no. 3, pp. 1140-1153, 2011.

[22] O. E. Rössler, "An equation for continuous chaos," Physics Letters A, vol. 57, no. 5, p. 397, 1976.

[23] O. E. Rössler, "An equation for hyperchaos," Physics Letters A, vol. 71, no. 2-3, pp. 155-157, 1979. 


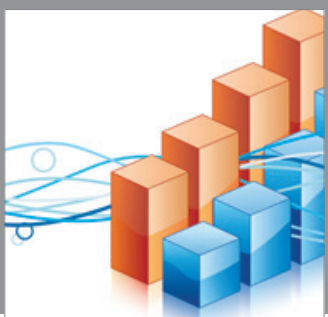

Advances in

Operations Research

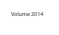

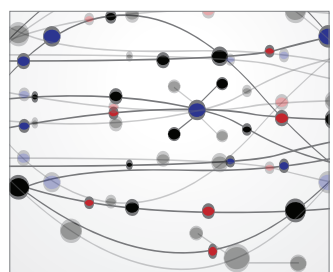

\section{The Scientific} World Journal
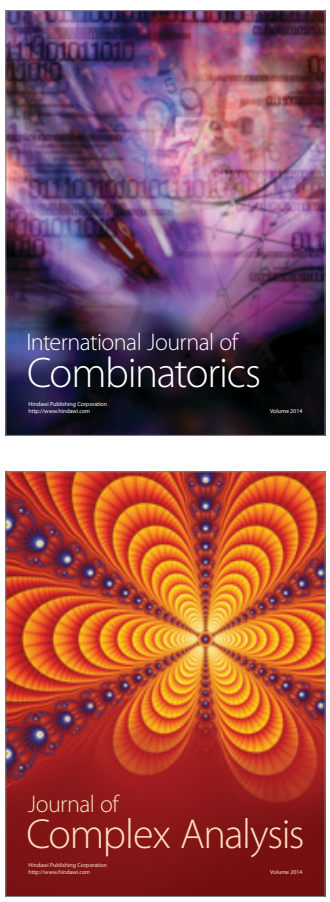

International Journal of

Mathematics and

Mathematical

Sciences
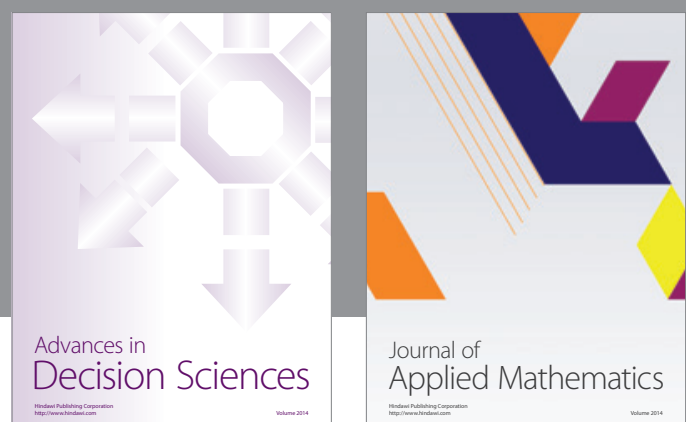

Journal of

Applied Mathematics
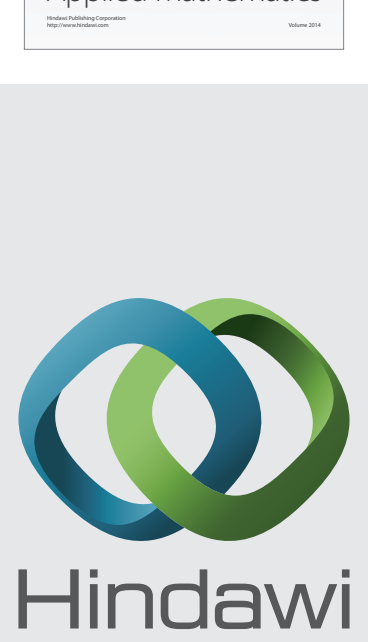

Submit your manuscripts at http://www.hindawi.com
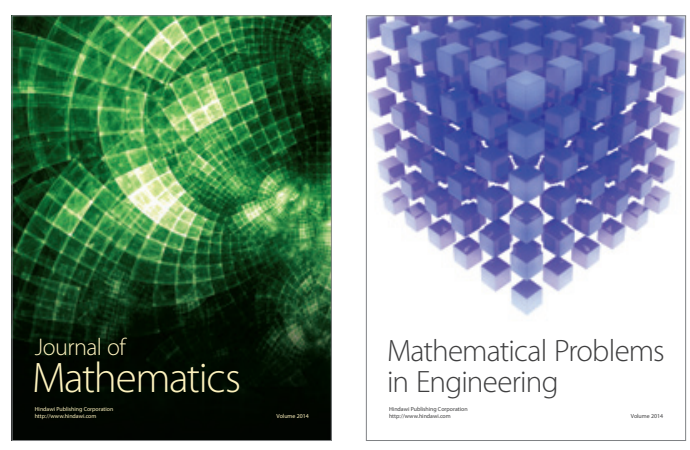

Mathematical Problems in Engineering
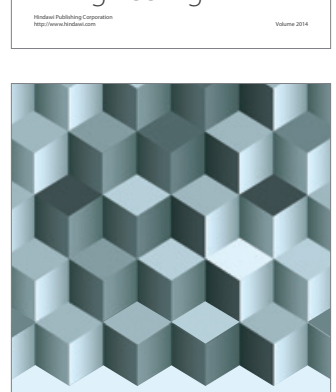

Journal of

Function Spaces
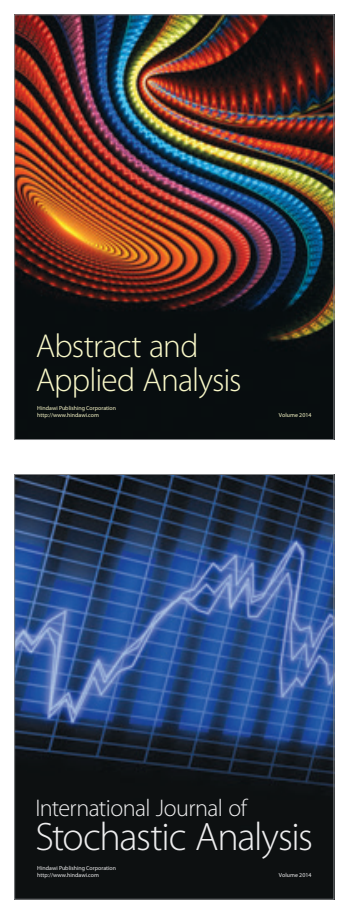

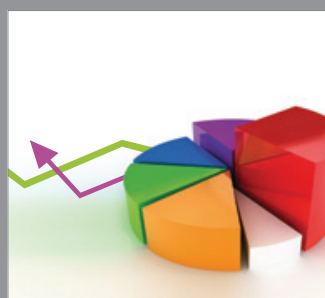

ournal of

Probability and Statistics

Promensencen
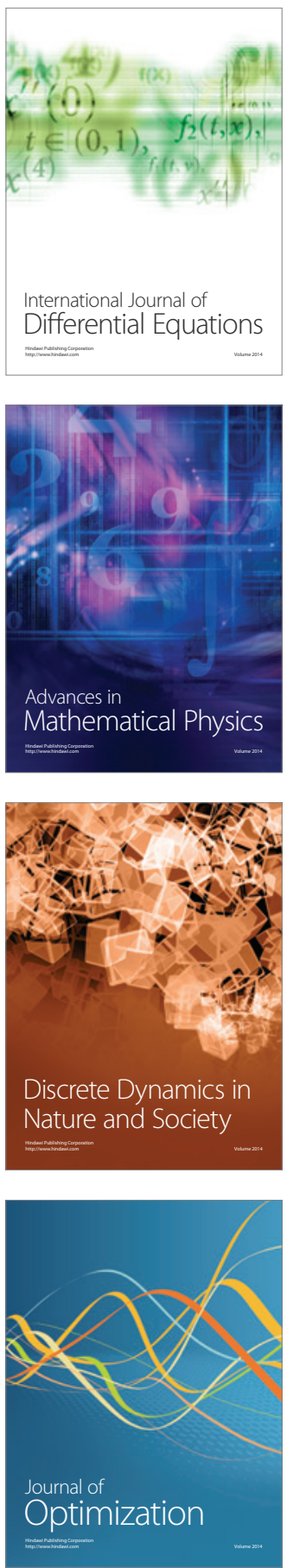